\title{
Impairments in the learning and performance of a new manual skill in patients with Parkinson's disease
}

\author{
CD FRITH, CA BLOXHAM, KN CARPENTER \\ From the Division of Psychiatry, MRC Clinical Research Centre, Harrow, UK
}

SUMmary Twelve patients with Parkinson's disease learned two novel skills in which they had to track a target by moving a joystick. In task 1 they had to learn to anticipate the movements of a semi predictable target. In task 2 they had to learn a novel control system in which the movements of the joystick were mirror reversed in relation to the computer screen.On each task they performed two sessions of three minutes continuous practice separated by a 10 minute rest. In both tasks the patients performed much worse than the controls, but showed clear evidence of learning, particularly after the ten minute rest. Detailed examination of their performance suggested that the skill was becoming automatic, releasing attention for aspects of the task that could not be learned. The major difference from the controls appeared during the first minute of each practice session when the controls showed a marked improvement in performance while the patients did not. We suggest that this rapid but temporary improvement in performance reflects the acquisition of a motor "set" whereby existing motor programs or skills are modified to suit the task currently in hand. We concluded that patients with Parkinson's disease have difficulty in maintaining such sets.

A number of attempts have been made in recent years to characterise the impairments of motor control associated with Parkinson's disease in terms of models of control derived from the study of normal volunteers. Thus Flowers ${ }^{1}$ has suggested that patients with Parkinson's disease have a problem with "open loop" control, but not with "closed loop" control. Marsden ${ }^{2}$ has suggested that Parkinsonian patients have difficulty in the "automatic execution of learned motor plans". Bloxham et $a^{3}$ propose that these patients have difficulty in initiating a motor plan, but no difficulty in executing the plan once it has been initiated. Wing and Miller ${ }^{4}$ suggest that Parkinson's disease patients have a deficit in activating a preplanned movement when the trigger is provided internally. The implicit assumption of all these authors is that there should be in Parkinson's disease a discrete impairment in one of the components of motor control corresponding to the relatively discrete brain lesions found in this disease. However, while there is a wealth of detailed clinical description in the literature about the impairment of motor control in Parkinson's disease there are relatively few experimental studies. In particular there are few studies on the

Address for reprint requests: Dr CD Frith, CRC Division of Psychiatry, Harrow HAl 3UJ, UK.

Received 5 March 1985 and in revised form 9 October 1985. Accepted 20 October 1985 ability of Parkinson's disease patients to learn a novel motor skill. Marsden ${ }^{2}$ reports some unpublished data which suggests that, unlike normal controls, some Parkinson's disease patients fail to learn a tracking task when given information about target predictability. On the other hand, Day et al ${ }^{5}$ found that Parkinson's disease patients selected to have no cognitive abnormalities did show an improvement in performance (decreased lag) when made aware that a target was moving predictably. This suggests that some degree of learning was taking place. In this paper we shall describe the learning and performance of two pursuit tracking tasks by Parkinson's disease patients and normal controls. In one task the movement of the target was partially predictable while in the other the control system was mirror reversed.

The learning of pursuit tracking tasks has been studied extensively since the $1920 \mathrm{~s}^{6}$ and a number of different aspects of the learning and performance of these tasks can be distinguished. Typically, with computer controlled equipment, the subject controls the position of the pointer on a screen by moving a joystick. His task is to keep the pointer as near as possible to a continuously moving target.

The most interesting paradigm for studying the learning of such a task is one in which short periods of work (circa 5 minutes) are separated by periods of rest (circa 10 minutes). Using this paradigm two phenom- 
ena can reliably be observed. ${ }^{6}$ (1) At the beginning of each session of work there is a rapid improvement of performance completed within 30 seconds. This effect is more marked in the later sessions of work. The size of this upswing is also dependent on the length of the preceding rest, being larger with longer rests. This then appears to be a temporary improvement in performance that is acquired at the beginning of each session of work and dissipates during rest. To distinguish it from permanent learning this phenomenon is often referred to as the acquisition of set. (2) After the initial upswing, performance within sessions tends to remain constant or even decline. However, after a rest there is a marked and permanent improvement in overall performance. This improvement from pre- to post-rest tends to be greater than the improvement during a session of continuous work. This post-rest improvement is known as reminiscence.

How these changes in performance relate to the underlying learning processes remains controversial. Following Fitts ${ }^{7}$ it is useful to distinguish three stages in the learning of a new skill. (1) In the cognitive stage the subject must acquire (implicitly or explicitly) the knowledge needed to perform the skill. Thus if target movement is predictable the subject must discover this by observing that the target always moves to position X2 after it has been in position $\mathrm{X} 1$ and so on. Having observed this he then knows that he can anticipate the position of the target by moving to position $\mathrm{X} 2$ when he sees the target in position X1 (thus achieving predictive performance using feedback). Similarly if his joystick control is mirror reversed he must discover that when the target moves to position $\mathrm{X} 1$ he must move the joystick to position $\mathrm{Xn}-1$. These items of knowledge are specific to the skill being learned. (2) In the strategic stage the subject must somehow use his knowledge to produce appropriate movements. Since, at least in the early stages of practice, the new skill is not yet available this must be done by modifying already existing skills (or motor programs) for this new purpose. At this stage performance will need much mental effort and conscious attention. (3) In the automatic stage the new skill has finally been learned. That is a specific motor program has developed for this skill and the subject no longer needs to modify programs from other skills. At this stage performance needs little mental effort or attention.

As a skill becomes automatic, performance of it interferes less with other tasks that are being performed at the same time. Frith and Lang ${ }^{8}$ exploited this prediction in order to study the development of automaticity in a tracking task. When both the horizontal and the vertical movements of the target were unpredictable then there was no improvement in performance. However, when one component was pre- dictable then (a) there was an improvement in the performance of this component during a session of work and (b) an improvement in the other unpredictable component after a rest. This result indicated that subjects had learned to track the predictable component. In addition, after rest, the tracking of this component had become more automatic and therefore interfered less with performance of the other, unpredictable component. The authors concluded that the phenomenon of reminiscence could be explained in terms of increased automaticity, while within sessions improvements could be interpreted in terms of strategic learning.

In the experiment reported here we have used Frith and Lang's methodology to investigate whether Parkinson's disease patients can (a) learn a new manual skill and (b) achieve a degree of automaticity in the performance of that skill. We investigated two kinds of learning using continuous tracking tasks. In one task (semi-predictable) the movements of the target were predictable in the horizontal dimension, but unpredictable in the vertical dimensions. Thus a subject could learn to predict the movements of the target in the horizontal dimension. This learning is of particular interest in relation to Parkinson's disease since Flowers ${ }^{1}$ has proposed that Parkinson's disease patients have difficulty in making anticipatory movements and can only follow a target using visual feedback to correct their errors. In the other task (mirror reversed) the movements of the target were unpredictable in both dimensions. However, the joystick control was mirror reversed so that in order to stay on target the subject had to move the joystick to the right when the target moved to the left. Thus, for the horizontal dimension, he had to learn to make a novel response to the visual feedback he received about errors. If Flowers is correct then Parkinson's disease patients should be less impaired on this mirror reversed task than on the semi-predictable one. It is possible to study within session (strategic) improvements in both tasks, but there is nothing in the literature to suggest whether or not Parkinson's disease patients should be impaired in this aspect of learning.

\section{Method}

Subjects had to track a target which moved in two dimensions on the face of a VDU with a maximum amplitude of $10 \mathrm{~cm}$. The target was a $4 \mathrm{~mm}$ square and the screen pointer was a cross which fitted exactly inside the square. The subject's task was to match the movement of the target as closely as he could, keeping the cross, if possible, inside the square. The position of the cross was controlled by means of a $17 \mathrm{~mm}$ long joystick which the subject held in his preferred hand. The subject's elbow rested on a foam rubber pad and 
Table 1 Track components $(\mathrm{Hz}$ )

\begin{tabular}{llll}
\hline & \multicolumn{3}{l}{$\begin{array}{l}\text { Semi-predictable } \\
\text { (task } 1 \text { l) }\end{array}$} \\
Horizontal & 0.30 & 0.30 & 0.30 \\
Vertical & 0.06 & 0.11 & 0.23 \\
& Mirror & reversed control & system \\
(task 2$)$ & & \\
Horizontal & 0.03 & 0.21 & 0.32 \\
Vertical & 0.05 & 0.19 & 0.33 \\
\hline
\end{tabular}

thus controls of the joystick required movements of the wrist and forearm.

Control of target movement Movement of the target in the vertical and horizontal directions was controlled independently. For each of these directions of target movement the position of the target every $25 \mathrm{~ms}$ was determined from a combination of sine waves.

Task 1 The horizontal component of this task was a single sine wave of $0.3 \mathrm{~Hz}$. It was thus repetitive and predictable. The vertical component of this task was formed from a combination of three sine waves of unrelated frequencies (see table 1). Thus this component was irregular and unpredictable. This task is essentially the same as the task II used by Frith and Lang ${ }^{8}$ except that the frequencies are slower and thus the task is easier and more suitable for older subjects. Task 2 In this task both the horizontal and the vertical components were composed from three unrelated sine waves (see table 1) and so all aspects of target movement were unpredictable. In addition the relation between joystick and screen pointer was reversed for the horizontal direction so that the movements of the joystick to the left caused the cross on the screen to move to the right. For vertical movements of the joystick the conventional relationship was maintained. Since this "mirror reversed" task is more difficult slower frequencies of target movement were used than in task 1. We aimed to choose a level of difficulty in both tasks that enabled subjects to achieve in the region of $50 \%$ time-on target, since this level of performance maximises the discriminating power of the measure.

Recording of tracking response Two voltages indicating the horizontal and vertical position of the joystick were fed into a PDP-11 computer via A/D converters and these controlled the position of the screen pointer (cross). The computer also controlled the position of the target and recorded the position of target and joystick every $25 \mathrm{~ms}$.

Procedure Subjects were first shown how movement of the joystick could control the position of the cross on the screen.
Once they had understood this control system and had found a comfortable position for their arm, subjects performed the semi-predictable task (task 1). Subjects were not told that target movements were predictable. Each subject performed the task continuously for three minutes. They then had a ten minute rest after which they again performed the task continuously for three minutes. After completing this task they were asked if they noticed anything regular or predictable in the target movement.

Subjects then performed a reaction time task ${ }^{9}$ for about 15 minutes. After this they performed the mirror reversed tracking task. They were told that the effect of moving the joystick had been altered and were shown how the new control system worked. Once they had understood this they performed two sessions of 3 minutes practice separated by a 10 minute rest.

\section{Analysis of performance}

Performance in the horizontal and the vertical directions was recorded separately. In each 30 seconds the total time for which the subject was within the target limits was recorded. (Obviously, since the two directions were considered independently, a subject would be considered on target vertically if he was within the limits of the vertical direction even if he was a long way from the target in the horizontal direction.) The two occasions of practice were divided into six 30 second trials. A rapid increase in performance at the very beginning of practice is supposed to reflect the acquisition of set. Given the 30 second measurement periods the earliest and shortest rise in the study is that between the first and second 30 second period. We therefore chose the difference between the first and second trials as our measure of "set". Additional within-session learning of the task was indicated by the increase that occurred during trials 2 to 6 . Overall performance on trials 2 to 6 was used to compare the first and second sessions of practice to obtain an estimate of between-sessions improvements. Within and between group comparisons were made using repeated measures Analysis of Variance, with appropriately adjusted degrees of freedom.

\section{Subjects}

Twelve patients with Parkinson's disease were tested. There were five women and seven men aged between 44 and 79 years. They were all outpatients with a history of the disease lasting at least 2 years. Details of the symptoms and the treatment regime at the time of testing are shown in table 2. None of these patients showed any evidence of cognitive impairment.

Table 2 Clinical status of the Parkinsonian group

\begin{tabular}{|c|c|c|c|c|c|c|c|c|}
\hline Subject & Sex & Age (yr) & Duration & Tremor & Rigidity & Bradykinesia & Social & Drugs \\
\hline $\begin{array}{r}1 \\
2 \\
3 \\
4 \\
5 \\
6 \\
7 \\
8 \\
9 \\
10 \\
11 \\
12\end{array}$ & $\begin{array}{l}\mathbf{F} \\
\mathbf{F} \\
\mathbf{F} \\
\mathbf{F} \\
\mathbf{F} \\
\mathbf{M} \\
\mathbf{M} \\
\mathbf{M} \\
\mathbf{M} \\
\mathbf{M} \\
\mathbf{M} \\
\mathbf{M}\end{array}$ & $\begin{array}{l}45 \\
66 \\
71 \\
72 \\
73 \\
44 \\
45 \\
53 \\
57 \\
59 \\
61 \\
79\end{array}$ & $\begin{array}{c}3 \cdot 5 \\
6 \\
6 \\
13 \\
3 \\
4 \\
3 \\
2 \\
6 \\
10 \\
4 \\
15\end{array}$ & $\begin{array}{l}\text { L severe } \\
\mathbf{L}+\mathbf{R} \text { slight } \\
\text { nil } \\
\mathbf{L}+\mathbf{R} \text { mod } \\
\mathbf{L}+\mathbf{R} \\
\text { nil } \\
\mathbf{L} \text { fine } \\
\mathbf{L} \text { mild } \\
\mathbf{L}+\mathbf{R} \text { mild } \\
\mathbf{R} \text { mild } \\
\mathbf{L}+\mathbf{R} \\
\mathbf{L}+\mathbf{R} \text { marked }\end{array}$ & $\begin{array}{l}\mathbf{L} \text { arm } \\
\mathbf{R}>\mathbf{L} \text { mod. } \\
\mathbf{L} \\
\mathbf{L}+\mathbf{R} \\
\mathbf{L}+\mathbf{R} \\
\mathbf{L} \text { marked } \\
\mathbf{L}+\mathbf{R} \bmod . \\
\mathbf{L}+\mathbf{R} \\
\mathbf{L}+\mathbf{R} \bmod . \\
\mathbf{L}+\mathbf{R} \\
\mathbf{L}+\mathbf{R} \bmod . \\
\mathbf{L}+\mathbf{R} \operatorname{marke}\end{array}$ & $\begin{array}{l}\mathbf{L} \text { arm } \\
\mathbf{L}+\mathbf{R} \text { mod. } \\
\text { nil } \\
\mathbf{L}+\mathbf{R} \text { mild } \\
\mathbf{L}+\mathbf{R} \\
\mathbf{L} \\
\mathbf{L} \text { mild } \\
\mathbf{L} \text { mod. } \\
\mathbf{L}+\mathbf{R} \text { mod. } \\
\mathbf{L}+\mathbf{R} \text { mod } \\
\mathbf{R} \text { marked } \\
\mathbf{L}+\mathbf{R} \text { marke }\end{array}$ & $\begin{array}{l}\text { ind. } \\
\text { dep. slight } \\
\text { ind. } \\
\text { ind. } \\
\text { ind. } \\
\text { ind. } \\
\text { ind. } \\
\text { ind. } \\
\text { ind. } \\
\text { ind. } \\
\text { ind. } \\
\text { d dep. mod. }\end{array}$ & $\begin{array}{l}\text { Sinemet } \\
\text { Artane Symmetrel } \\
\text { Sinemet } \\
\text { Sinemet Kemadrin } \\
\text { nil } \\
\text { Sinemet } \\
\text { Lorazepam } \\
\text { Sinemet } \\
\text { Sinemet } \\
\text { Sinemet Artane } \\
\text { Sinemet Disipal } \\
\text { Sinemet }\end{array}$ \\
\hline
\end{tabular}


All the patients had been contacted through the local Parkinson's Disease Society and had volunteered to take part in the experiment. Nine were right handed and three were left handed. They used their preferred hand to perform the tracking task. One subject found the semi-predictable tracking task (task 1) too difficult (less than 5\% time on target) and had to be excluded. For the same reason four subjects had to be excluded from the mirror reversed tracking task (task 2).

The control group consisted of 13 volunteers, seven women and six men, from the League of Friends and from ancilliary, scientific and technical staff at Northwick Park Hospital. They were aged between 44 and 75 years. All but two controls were right handed and they used their preferred hand to perform the tracking tasks. No control subject had to be excluded for poor performance.

Informed consent was obtained from every subject.

\section{Results}

Task 1: Semi-predictable tracking test

Figures 1 and 2 show the results for the semi-

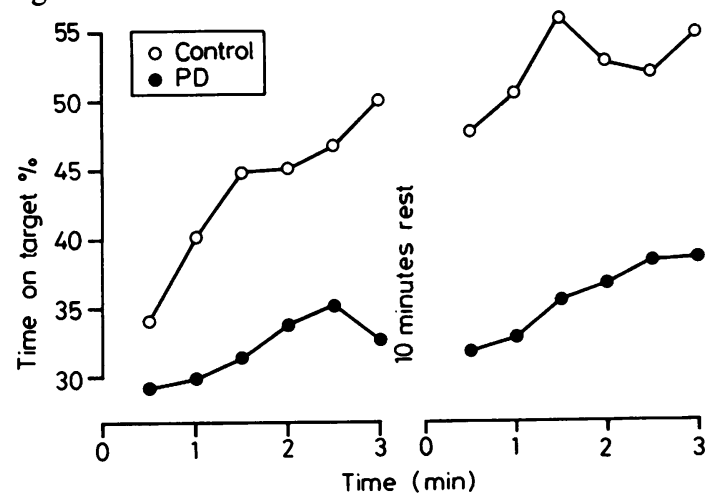

Fig 1 Mean time on target for the predictable (horizontal) component of task 1 (semi-predictable target movement). ( $P D=$ Parkinson's disease patients.)

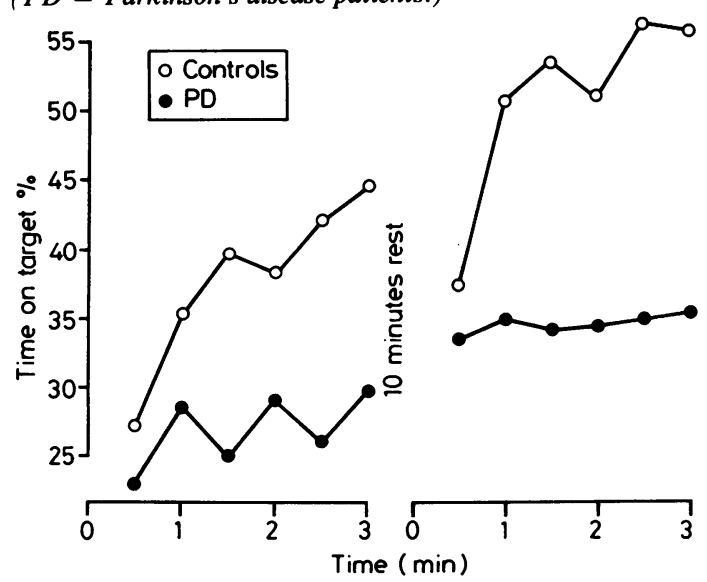

Fig 2 Mean time on target for the unpredictable (vertical) component of task 1 (semi-predictable target movement). predictable track; fig 1 showing the predictable horizontal component and fig 2 the unpredictable vertical component.

The overall impression is that the control group perform better and learn faster than the Parkinson's disease group, particularly during the first two trials of each occasion. ANOVA revealed a highly significant improvement in performance from trial 1 to $2(\mathrm{~F}(1,20)=19.8, \mathrm{p}<0.0001)$. However this increase was significantly greater in the control group $(F(1,20)=8.36, p<0.01)$. There was no significant improvement in the Parkinson's disease patients $(F(1,9)=0.88)$.

There was a highly significant improvement during trials 2 to 6 (linear $F(1,20)=14.6, p<0.001)$. There was also a significant interaction with direction such that improvement was greater for the horizontal, predictable component than for the vertical unpredictable component (linear $F(1,20)=5.49, p<0.05)$. There were no significant differences between the groups in this aspect of learning. When analysed separately the Parkinson's disease patients showed a significant improvement in performance (linear $\mathrm{F}(1$, $9)=6.51, \mathrm{p}<0.05$ ). There was a very highly significant difference between the two sessions of practice $(F(1,20),=35 \cdot 1, p<0.0001)$. This improvement was the same for both directions, but was significantly greater in the control group (F (1, 20) $=5.98, p<0.05)$. Nevertheless the Parkinson's disease patients did also show an improvement across sessions for the unpredictable, vertical component $(F(1,9)=5.94, p<0.05)$. No one in the control group and only one member of the patient group reported noticing anything predictable or regular in the movement of the target.

\section{Task 2: Mirror reversed}

Figures 3 and 4 show the results for the mirror reversed track; fig 3 showing the mirror reversed horizontal component and fig 4 the non-reversed vertical component. Both components were unpredictable. Once again the controls performed better and showed much faster learning. The improvement shown by the controls during the first minute of practice was even more striking than that shown for the semipredictable task. There was a highly significant improvement in performance from trials 1 to $2(\mathrm{~F}(1$, $17)=12.5, \mathrm{p}<0.01)$. This was significantly greater in the controls $(F(1,17)=8.03, p<0.02)$. There was no significant improvement in the Parkinson's disease patients $(F(1,6)=0 \cdot 30)$.

There was a small but significant improvement in performance within sessions from trials 2 to 6 (linear $\mathrm{F}(1,17)=4.84, \mathrm{p}<0.05)$. However there was also a significant interaction between groups, directions and trials (linear $F(1,17)=5 \cdot 18, p<0 \cdot 05$ ). This was 


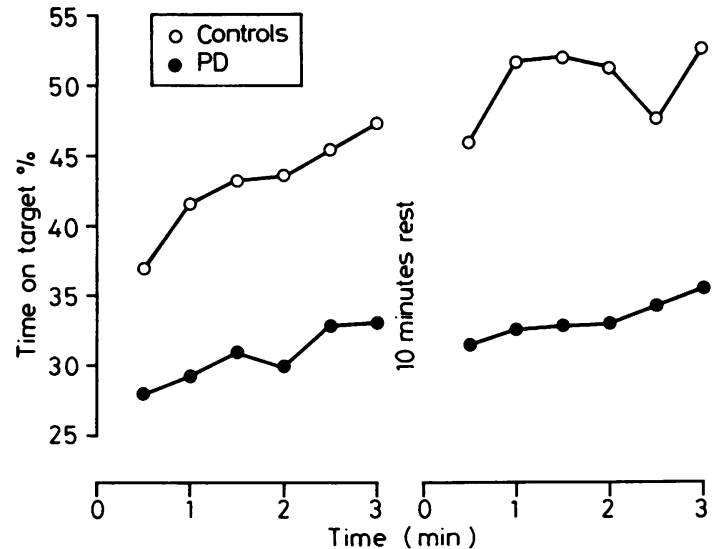

Fig 3 Mean time on target for the reversed (horizontal) component of task 2 (mirror tracking).

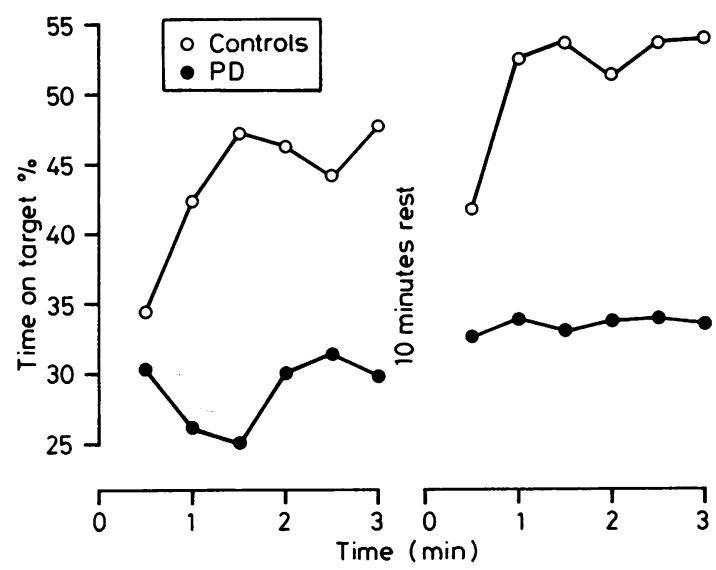

Fig 4 Mean time on target for the normal (vertical) component of task 2 (mirror tracking).

because the control group showed an improvement for the horizontal, reversed component, but not for the vertical component, while the Parkinson's disease group showed an improvement in neither component $(F(1,6)=0.62)$. Thus in this task the Parkinson's disease patients showed no significant within session improvements. There was a highly significant improvement from one session to the next $(F(1,17)$ $=56.8, \mathrm{p}<0.0001)$. This improvement was slightly larger for the control group $(\mathrm{F}(1,17)=3 \cdot 16, \mathrm{p}<$ $0 \cdot 10)$. However the Parkinson's disease group showed a marked improvement in both components also ( $F$ $(1,6)=9.42, \mathrm{p}<0.05)$.

\section{Discussion}

The performance of the control group was essentially similar to that observed in other continuous tracking tasks. ${ }^{6}$ In both sessions of work there was a marked improvement during the first 30 seconds and rather less thereafter. This effect was more marked on the second session of work than the first. Performance after the rest, except for the very first trial, was markedly better than performance before the rest. The performance of the Parkinson's disease patients was clearly worse than that of the controls, but also showed qualitative differences. Nevertheless, like the controls the patients showed significant between sessions improvements for both tasks and, in the case of the mirror tracking task, this improvement was only slightly and insignificantly less than the controls. However the patients showed almost no within session improvements. This lack of improvement was most striking for the first two trials of each practice session during which time the patients showed no significant improvement on either task. For the 3 minute session as a whole the patients showed no improvement on the mirror tracking task, but a slight improvement on the semi-predictable task.

\section{Temporary and permanent learning}

In the introduction we suggested that between sessions improvements reflect permanent learning whereas within session improvements reflect temporary learning. The results of the present experiment suggest that Parkinson's disease patients are relatively unimpaired in the permanent component of skill learning, but are markedly impaired in the temporary component. The consequences of this defect are particularly striking in the mirror tracking task (figs 3 and 4). On the first 30 second trial after the rest the performance of the controls was much worse than on the last 5 pre-rest trials. Presumably this is because the temporary learning acquired during the first session of work has been lost during the rest. In striking contrast the first post-rest trial of the Parkinson's disease patients was much better than any pre-test trial. We suggest that since the patients did not acquire a temporary component of learning during the pre-rest work they did not have any to lose during the rest. Thus only the increment in performance due to permanent learning is revealed.

A different pattern is found for the semipredictable task. While the controls do show more improvement than the Parkinson's disease patients at the very beginning of practice, both groups show within session improvements thereafter. After rest there is a slight decrement in performance for both groups also. There is a major difference between the two tasks in terms of the availability of the knowledge necessary for acquiring the skill. In the mirror tracking tasks subjects were told, and indeed it would become obvious to them at the very beginning of practice, that the joystick control had been reversed 
so that if the target moved to the left they would have to move to the right. Thus they could attempt to use this knowledge immediately. In the semi-predictable task the knowledge that the target moved regularly in the horizontal direction was not given to the subjects and few of them became aware of it during practice. The slow within session improvements shown by both groups might reflect the gradual, and unconscious, acquisition of this knowledge. This difference between the tasks might imply that controls can make better use of explicitly given knowledge about the task than can patients with Parkinson's disease. This hypothesis has been directly investigated by Bronstein and Kennard ${ }^{10}$ who studied the tracking of a predictable target by eye. When subjects were unaware of the predictable nature of the target movement there was little difference between Parkinson's disease patients and controls and both showed a steady improvement in performance. However, when the information about predictability was given the performance of the controls altered far more than that of the Parkinson's disease patients in that they produced many more anticipatory responses.

\section{The automatisation of learning}

We have suggested that the permanent, between sessions improvements in performance reflect the automatisation of the skill being learned, particularly if it occurs in the unpredictable components of the task. From this point of view our Parkinson's disease patients were acquiring a new skill that they could perform automatically on both tasks. Crucial to this argument is the claim that little or no learning is possible for an unpredictable component of a task, so that any improvement must be due to reduced attention to other components. This claim was justified in Frith and Lang's study ${ }^{8}$ since no improvement occurred on an unpredictable version of the task used. However, the tasks used in the present study were deliberately made easier (by using slower target frequencies) to suit less able subjects. Clearly, although the movements of the target were unpredictable in the long term, short term predictions were possible particularly if the target was moving relatively slowly. For short periods the target will be moving in a constant direction at a constant speed and the subject could learn something about the average speed of the target. In order to check this possibility we tested additional controls (matched for age) on unpredictable versions of the tasks with various average target speeds. In these tasks the target moved unpredictably in both directions and control was not mirror reversed. We found that there were small between sessions improvements for these tasks particularly for those in which the average target speed was low. However the improvements were far less than those shown by the patients and controls in the experimental tasks. For example in the mirror tracking task controls and Parkinson's disease patients improved by $18 \%$. In a slow unpredictable task (which was easier than in the mirror tracking task in terms of time on target) between sessions improvement was $10 \%$ and in fast unpredictable tasks (which was more difficult than the mirror tracking task) improvement was only $6 \%$. We would conclude that there was evidence from our data that patients with Parkinson's disease can learn a new skill to a level at which it can be performed to some extent automatically.

\section{The nature of the temporary improvement in performance (Set)}

The major abnormality shown by the Parkinson's disease patients on these tasks was a lack of any improvement in performance during the first $30 \mathrm{sec}-$ onds of practice in both the first and the second session of work. We have argued above that this improvement reflects some temporary learning process that might be labelled "set". What is it then that normal people learn so rapidly at the beginning of a practice session, but then forget during the rest?

One possibility is that "set" reflects the adaptation of the subject to non-specific features of the task. It would be desirable that these aspects of performance be acquired only temporarily since they may be modified from one session to the next. For example the precise way in which limbs and muscles have to be controlled to produce the required movement of the joystick will depend on how the joystick is held, the position of the elbow and so on. At the very beginning of practice the subject will have to adjust his motor programs to allow for these variations. In some circumstances these variations can be extreme. For example holding the steering wheel of a car in the 6 o'clock position with one hand requires very different movements from the $\mathbf{1 0}$ to 2 position with two hands. Nevertheless a driver can adjust to these variations with ease.

A contrasting possibility is that the adjustment is in relation to features specific to the task being learned. For example in a choice reaction time task the relations between stimulus and response are usually arbitrary, for example lifting the left index finger when the red light goes on and the right finger when the green light goes on. To perform this task the subject must temporarily "attach" his left finger lifting program to the appearance of a red light. Such temporary attachments are commonly required in real life situations. They can be temporary since the same task may only be performed once. If it is performed repeatedly then special programs will develop and performance becomes skilled. The same mechanism could apply to the continuous tracking tasks used in 
this experiment. In the mirror reversed task for example the subject must attach his program for moving the joystick to the left to the stimulus of the target moving to the right. In the semi predictable task he must attach his program for moving the stick to position X2 to the stimulus of the target being in position $\mathrm{X} 1$. It seems plausible that these temporary attachments would be made at the very beginning of practice, if the necessary knowledge were available and that the rapid improvement of performance at this time would reflect the success of these attachments.

These two possibilities can easily be distinguished since, if set reflects adaptation to non specific features of the task then it should occur even when there is nothing new in the task to be learned. We were able to examine this in the new control data collected from the tasks in which the target movements were unpredictable and the joystick control was not mirror reversed. The results were very clear cut. In such tasks there was little or no increase at the very beginning of a practice session. Thus the rapid post-rest upswing we found in the experimental tasks does not reflect adaptation of the subject to non-specific features of the task. Thus the appearance of post-rest upswing seems to be associated with the novel features that have to be learned in a task. We suggest that upswing reflects the success with which the subject can make novel use of existing motor programs on the basis of the knowledge he has about the task requirements. This can be seen as a coping strategy to be used while new motor programs are developed that are specific to the novel task. The observation that upswing occurs even for the component in the task for which there are no novel features to be learned suggests that, once the appropriate set has been developed it can be maintained with little attention.

On this account patients with Parkinson's disease, while they can develop new motor programs for a novel task, have difficulty in deploying already existing programs in novel situations.

\section{Implications for models of Parkinsonian deficit}

(1) Flowers ${ }^{1}$ has suggested that Parkinson's disease patients have a specific difficulty with "open loop" control of movement. In other words they cannot anticipate the movement of a target, but must follow it. We modify this proposal by suggesting that while Parkinson's disease patients cannot use their knowledge of the target movement to produce anticipatory movements, they can eventually learn the skill of making anticipatory movements in a particular task. Thus Parkinson's disease patients will be excessively reliant on feedback in the early stages of performance in a novel task. This account may explain why some studies do find evidence of open loop control in Parkinson's disease patients ${ }^{35}$ while others do not. ${ }^{1}$

(2) Bloxham et al $^{3}$ suggest that the problem resides in the initiation of preprogrammed movements. We would now modify this formulation and suggest that initiation difficulties are particularly likely when the movement involves the deployment of a motor program in novel circumstances. This would be the case when the movement is elicited by a stimulus different from the one for which it was developed.

(3) Wing and Miller ${ }^{4}$ suggest that the difficulty lies in using an internal trigger to initiate a preprogrammed movement. This would be consistent with our account if an internal trigger is involved whenever a program has to be initiated in a novel manner. That is, not only in situations where there is no specific external stimulus, but also when the program is to be initiated by a stimulus other than the one for which it was originally developed. In this latter case there would presumably have to be some intermediate link between the stimulus and the program, hence the internal nature of the trigger.

(4) Marsden ${ }^{2}$ proposes that the problem lies in the "automatic execution of learned motor plans". We believe our results show that Parkinson's disease patients can perform skilled movements automatically. However Marsden (personal communication) implies something more complex by his formulation, that is the ability to link together smoothly a number of different motor programs. This would be consistent with our formulation if the sequence required was a novel one. However, we would expect that patients would have little difficulty with a much practised sequence.

\section{Some wider aspects of set}

We have suggested that the development of motor set may be defined as the modification of an existing motor program for use in a new situation. In the forewarned or cued reaction time task the advantage resulting from prior information occurs because the subject can prepare the movement (that is, modify the program) in advance of the stimulus. Woodworth ${ }^{11}$ called this preparation the development of muscular set. Thus the observation by Evarts et al $^{12}$ and Bloxham et $a l^{3}$ that Parkinson's disease patients fail to gain advantage from advance information in a choice reaction time task can also be explained in terms of a failure to develop an appropriate motor set. DennyBrown and Yanagisawa ${ }^{13}$ have suggested that the basal ganglia are particularly concerned in activating "set" or "pump priming" for a certain act, that is the preparation of the mechanism preparatory to a motor performance orientated to the environment.

Set in the sense discussed above need not be restricted to motor behaviour, but could also apply to thinking and problem solving. The poor performance 
of Parkinson's disease patients on the Wisconsin card sorting test ${ }^{14}$ could be seen as due to a problem with set in this sense. Flowers and Robertson ${ }^{15}$ demonstrated poor performance in Parkinson's disease patients on a simplified form of the Wisconsin task which they suggest reflects more directly the difficulty these patients have in maintaining a mental set. In this task the subject has to continually modify the strategy he uses for solving a problem by attending to different dimensions of stimuli on alternate trials. Thus the mental set required for this task is clearly similar to the motor set we have suggested is involved in tracking. Both kinds of set seem to be impaired in patients with Parkinson's disease.

This study was conducted under the auspices and according to the rules of the Ethical Committee of Northwick Park Hospital. We are grateful to Julie Keenan for collecting the additional control data.

\section{References}

${ }^{1}$ Flowers K. Lack of prediction in the motor behaviour of Parkinsonism. Brain 1978;101:35-52.

${ }^{2}$ Marsden CD. The mysterious motor function of the basal ganglia: The Robert Wartenberg lecture. Neurology (NY) 1982;32:14-39.

${ }^{3}$ Bloxham CA, Mindel TA, Frith CD. Initiation and execution of predictable and unpredictable movements in Parkinson's disease. Brain 1984;107:371-84.

${ }^{4}$ Wing A, Miller E. Basal ganglia lesions and psychological analyses of voluntary movement control. CIBA Symposium 1984;107.

${ }^{5}$ Day BL, Dick JPR, Marsden CD. Patients with Parkinson's disease can employ a predictive motor strategy. $J$ Neurol Neurosurg Psychiatry 1984;47:1299-306.

${ }^{6}$ Eysenck HJ, Frith CD. Reminiscence, Motivation and Personality. New York: Plenum Press, 1977.

${ }^{7}$ Fitts P. Factors in complex skill training. In: Glaser R, ed. Training Research and Education. University of Pittsburgh Press, 1962.

${ }^{8}$ Frith $C D$, Lang $R$. Learning and reminiscence as a function of target predictability in a two-dimensional tracking task. $Q J$ Exp Psychol 1979;31:103-9.

${ }^{y}$ Scott KN. The effects of advance information on initiation and execution of movements in Parkinson's disease. Unpublished research dissertation for the BPS Diploma in Clinical Psychology, 1983.

${ }^{10}$ Bronstein AM, Kennard C. Predictive ocular motor control in Parkinson's disease. Brain (in press).

${ }^{11}$ Woodworth RS. Experimental Psychology. New York: Holt, 1938.

12 Evarts EV, Teravainen H, Calne DB. Reaction time in Parkinson's disease. Brain 1981;104:167-86.

${ }^{13}$ Denny-Brown D, Yanagisawa N. The role of the basal ganglia in the initiation of movement. In: Yahr MD, ed. The Basal Ganglia. New York: Raven Press, 1976:115-49.

${ }^{14}$ Bowen FP. Behavioural alterations in patients with basal ganglia lesions. In: Yahr MD, ed. The Basal Ganglia. New York: Raven Press, 1976:169-80.

${ }^{15}$ Flowers KA, Robertson C. The effect of Parkinson's Disease on the ability to maintain a mental set. $J$ Neurol Neurosurg Psychiatry 1985;48:517-29. 